\title{
Escherichia coli Nissle 1917 bacterial ghosts retain crucial surface properties and express chlamydial antigen: an imaging study of a delivery system for the ocular surface
}

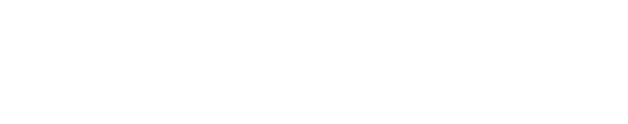

\author{
Jacqueline Montanaro' \\ Aleksandra Inic-Kanada' \\ Angela Ladurner' \\ Elisabeth Stein' \\ Sandra Belij' \\ Nora Bintner' \\ Simone Schlacher' \\ Nadine Schuerer' \\ Ulrike Beate Mayr ${ }^{2}$ \\ Werner Lubitz ${ }^{2}$ \\ Nikolaus Leisch ${ }^{3}$ \\ Talin Barisani-Asenbauer' \\ 'Laura Bassi Centres of Expertise, \\ OCUVAC - Centre of Ocular \\ Inflammation and Infection, Centre \\ for Pathophysiology, Infectiology, \\ and Immunology, Medical University \\ of Vienna, Vienna, Austria; ${ }^{2}$ BIRD-C \\ GmbH \& Co KG, Kritzendorf, Austria; \\ ${ }^{3}$ Department of Ecogenomics and \\ Systems Biology, University of Vienna, \\ Vienna, Austria
}

\begin{abstract}
To target chronic inflammatory ocular surface diseases, a drug delivery platform is needed that is safe, possesses immunomodulatory properties, and can be used either for drug delivery, or as a foreign antigen carrier. A new therapeutic approach that we have previously proposed uses nonliving bacterial ghosts (BGs) as a carrier-delivery system which can be engineered to carry foreign antigens and/or be loaded with therapeutic drugs. The parent strain chosen for development of our BG delivery system is the probiotic Escherichia coli strain Nissle 1917 (EcN), whose intrinsic properties trigger the innate immune system with the flagella and fimbriae used to attach and stimulate epithelial cells. In previous studies, we have shown that EcN BGs are safe for the ocular surface route, but evidence that EcN BGs retain flagella and fimbriae after transformation, has never been visually confirmed. In this study, we used different visualization techniques to determine whether flagella and fimbriae are retained on EcN BGs engineered either for drug delivery or as a foreign antigen carrier. We have also shown by immunoelectron microscopy that $\mathrm{EcN}$ retains two foreign antigens after processing to become EcN BGs. Furthermore, we demonstrated that BGs derived from EcN and expressing a foreign antigen attachment to conjunctival epithelial cells in vitro without causing reduced cell viability. These results are an important step in constructing a delivery system based on a nonliving probiotic that is suitable for use in ocular surface diseases pairing immunomodulation and targeted delivery.
\end{abstract}

Keywords: delivery system, flagella, fimbriae, foreign antigen, electron microscopy, epithelial cells

\section{Introduction}

Chronic ocular surface diseases pose serious risks of sequelae that harm vision. For example, the combined chronic inflammation symptoms of blurred vision, painful, itchy, swollen eyelids, and dry or red eyes could eventually progress to cause corneal scarring and blindness. Recent studies suggest that scarring damage at the ocular surface may be due to the immune response to infections or allergens. ${ }^{1}$ Thus, new treatments to control the immune response for ocular surface diseases aim to mitigate and control inflammation and thereby reduce the risk of vision loss sequelae. Recently, the beneficial effects of treatment with eye drops containing live probiotic bacteria were described on a patient suffering from allergic vernal keratoconjunctivitis. ${ }^{2}$

The use of probiotics for the treatment of inflamed gut mucosa is already well established, but these are still live bacteria. ${ }^{3,4}$ We have concerns that live probiotic bacteria may not be sufficiently safe to use in eye drop applications because when live
Correspondence: Talin BarisaniAsenbauer

Laura Bassi Centres of Expertise, OCUVAC - Centre of Ocular Inflammation and Infection, Centre for Pathophysiology, Infectiology, and Immunology, Medical University of Vienna, Sensengasse 2a, A-1090 Vienna, Austria

Tel +43 I 4016033150

$\mathrm{Fax}+43$ । 40 I6093 8293

Email talin.barisani@meduniwien.ac.at 
bacteria are applied to inflamed ocular mucosa, there is still a risk of bacterial multiplication, infection, and horizontal gene transfer to, or from, resident conjunctival flora. Therefore, we aimed to find a safer alternative to probiotic live bacteria, which could be used topically for immunomodulation and drug delivery to the ocular surface.

Bacterial ghosts (BGs) are a nonliving alternative to live probiotics for ocular surface treatment and drug delivery. They are the nonliving membrane shells of Gram-negative bacteria without their cytoplasmic and DNA content, formed during exponential growth when a change in temperature induces activation of the $E$-lysis plasmid. In response to $\mathrm{E}$ plasmid expression, the inner and outer membranes fuse to form a trans-membrane tunnel in the cell wall, allowing for the expulsion of the cytoplasm and DNA contents while leaving the bacterial cell wall intact. Thus, the BG remains as a hollow sac, which can be filled with drug compounds ${ }^{5,6}$ or prior to $E$-lysis, the bacterial cell wall can be altered to express foreign proteins. ${ }^{7,8}$ As the bacterial membrane of BGs remains intact, it is assumed that all of the outer surface structures, such as the fimbriae and flagella, also remain intact. Nevertheless, the large-scale production of BGs in liquid culture requires active aeration and agitation, conditions in which flagella are likely to shear or break off the cells. ${ }^{9}$ Such potential problems have not been investigated by direct microscopic methods.

Building on the idea of using BGs as an immunomodulatory ocular surface disease treatment, we chose an established probiotic bacterium (Escherichia coli strain Nissle 1917 [EcN]) for making BGs. EcN has been used for nearly 100 years in both human and veterinary medicine as a safe probiotic for the treatment and immunomodulation of various autoinflammatory diseases. ${ }^{10}$ The special advantage of EcN BGs for eye drop delivery over alternatives would be that they could act as both a drug-delivery system and immune modulators. Theoretically, the hollow EcN BG sac could be filled with antibiotics, whereas the outer membrane expresses immunomodulatory foreign proteins. Nevertheless, it is important to establish first whether EcN BGs retain their outer membrane structures, such as fimbriae and flagella. These outer morphological structures carry pathogen-associated molecular patterns that bind to toll-like receptors (TLRs) on cells of the immune system and were proven to be necessary for live $\mathrm{EcN}$ to activate innate immune responses. These beneficial immunomodulatory effects ${ }^{11-13}$ could contribute to the prevention of ocular surface diseases. ${ }^{5,14}$

To fight human ocular surface diseases, the stimulation of humoral secretory immunoglobulin A and cellular interferon gamma secretion are crucial for mucosal immune modulation. ${ }^{15}$ BGs can carry foreign immune-stimulatory antigens on their surface to reduce or modulate disease symptoms. Because both native and foreign proteins can be expressed in the cell wall membrane envelope of BGs before E-mediated lysis, multiple antigens of various origins could be presented to the immune system simultaneously. ${ }^{16}$

The current challenge for the treatment of ocular surface diseases lies in the development of safe and effective methods for carrier systems for both immunomodulation and drug delivery without active or passive transmission or translocation into the eye. ${ }^{17}$ In particular, we are developing a delivery-carrier system for the treatment or immunomodulation of trachoma, an ocular surface disease caused by Chlamydia trachomatis (CT). To develop the EcN BGs that could stimulate the immune response in trachoma, ${ }^{18-22}$ the major outer membrane proteins (MOMPs) and a polymorphic membrane protein $\mathrm{C}$ (PmpC) were shown to be expressed on probiotic EcN BGs. The broader concept, whether "armed"BGs could act as both an antigen carrier and delivery system and eventually have the potential to treat, immunomodulate, and prevent ocular surface diseases, was tested.

The purpose of this study was to examine whether EcN BGs preserve essential outer membrane structures, such as the flagella or fimbriae, and co-express foreign antigens (MOMP or PmpC) while retaining the capacity for attachment to conjunctival epithelial cells.

\section{Materials and methods Preparation of BGs}

Genomic DNA preparation and PCR amplification of foreign chlamydial antigen sequences

$\mathrm{N}-\mathrm{PmpC}$

The N-terminal part of the PmpC coding sequence was amplified from purified genomic DNA using a high-fidelity polymerase chain reaction (PCR) system (Fermentas, Fisher Scientific-Austria GmbH, Vienna, Austria) and oligonucleotide primers flanked with specific restriction sites. The primer design was based on a CT sequence obtained from a published data bank (Gene ID: 7882761). For N-PmpC amplification, the forward primer (pBGKB-N-PmpC fwd) incorporated a $K p n I$ restriction enzyme site with the sequence 5' ATA GGT ACC ATG AAG TTT ATG TCA GCT ACT GCT 3', and the reverse primer ( $\mathrm{pBGKB}-\mathrm{N}-\mathrm{PmpC}$ rev) incorporated an $\mathrm{Xba \textrm {I }}$ site $5^{\prime}$ ATA TCT AGA GT CCC GCT ATC GAG ATT AAA CAA 3'. The amplified PCR product (2,699 bp) was isolated from a $1 \%$ agarose gel and purified with a gel extraction kit (Thermo Fisher Scientific, Waltham, MA, USA). 


\section{MOMP}

The leaderless part of the MOMP coding sequence was amplified from purified genomic DNA using a high-fidelity PCR system (Fermentas, Fisher Scientific-Austria GmbH) and oligonucleotide primers flanked with specific restriction sites. The primer design was based on the CT sequence obtained from a published data bank (Gene ID: 7882602). For MOMP amplification, the forward primer (pBGKB-MOMP fwd) incorporated a $K p n I$ restriction enzyme site with the sequence $5^{\prime}$ TA TAT GGT AC'C C GAA GCG GAA TTG TGC ATT TAC 3', and the reverse primer (pBGKB-MOMP rev) incorporated a $B g l \mathrm{II}$ site $5^{\prime}$ TA TAT A'GA TCT CCT GTG GGG AAT CCT GCT 3'. The amplified PCR product (4,870 bp) was purified with a PCR Purification Kit (Thermo Fisher Scientific).

\section{Construction of the PBGKB-N-PmpC and pBGKB- MOMP vectors}

The $\mathrm{pBGKB}$ vector is based on the pBAD-gIII-B vector (Invitrogen, Life Technologies), with the ampicillin resistance cassette exchanged with a kanamycin resistance cassette. The plasmid contains a gIII signal sequence, which relies on the bacterial Sec transport mechanism to direct expressed proteins into the periplasmic space. A fusion protein is generated by cloning the foreign chlamydial protein (N-PmpC or MOMP) into the multiple cloning site of the $\mathrm{pBGKB}$ vector, which is followed by a c-Myc tag and polyhistidine tag for recombinant protein detection.

\section{Production of EcN BGs carrying chlamydial foreign antigen-gll-fusion proteins}

Competent $E$. coli Nissle (pBGKB-N-PmpC) and competent E. coli Nissle (pBGKB-MOMP) were produced independently using the following method: bacteria were transformed with the $E$-lysis plasmid pGLysivb, cells harboring a combination of lysis and expression plasmids were used for the production of N-PmpC and MOMP BGs. Transformants were grown at $36^{\circ} \mathrm{C}$ in lysogeny broth supplemented with kanamycin $(50 \mu \mathrm{g} / \mathrm{mL})$ and gentamicin $(100 \mu \mathrm{g} / \mathrm{mL})$ in a $20 \mathrm{~L}$ stirring batch fermenter (TECHFORS S1820 Infors AG, Bottmingen, Switzerland). When an optical density at $600 \mathrm{~nm}$ of 0.9 was reached, the expression of N-PmpC or MOMP fusion proteins was induced by the addition of $0.1 \%$ (pBGKB-N-PmpC) or $0.2 \%$ (pBGKB-MOMP) L-arabinose, and 10 minutes later, gene-E-mediated expression was induced by increasing the temperature from $36^{\circ} \mathrm{C}$ to $42^{\circ} \mathrm{C}$. To monitor the lysis efficiency, viable cell counts were determined using a spiral plater (WASP System, Don Whitley Scientific
Limited, Shipley, UK). Lysis reached 99.9\% efficiency after 120 minutes. After growth and lysis, the broth was harvested by tangential flow filtration $(0.2 \mu \mathrm{m})$ and transferred to a $\beta$-Propiolactone treatment flask to kill any remaining live bacteria $(<0.01 \%)$ and eliminate the DNA residue and any possibility of genetic material transfer. ${ }^{23}$ After washing, the BGs were processed as previously described. ${ }^{5}$

\section{Sample collection}

Samples were collected at the indicated time points during batch fermentation. They were centrifuged at 4,000× $g$, washed once with phosphate-buffered saline (PBS) to remove microbial growth media, and then centrifuged again. Pellets were immediately chemically fixed for immunohistochemistry and electron microscopy studies, or stored frozen for Western blot analysis.

\section{Western blot for foreign chlamydial antigens and flagella in $\mathrm{EcN}$}

Western blot was performed as previously described. ${ }^{24}$ Chlamydial proteins were co-expressed with the c-Myc tag under the same inducible promoter. Thus, the detection of c-Myc was inferred as foreign chlamydial protein expression. The membrane was probed with 1:500 anti-c-Myc antibody (BioLegend, San Diego, CA, USA) and 1:1,000 anti-mouse IgG-AP-linked antibody (Cell Signaling Technology, Inc., Danvers, MA, USA) as a secondary antibody. The intensity of the specific bands was visualized with 1-step NBT/BCIP (5-bromo-4-chloro-3-indolyl-phosphate/nitro blue tetrazolium) substrate solution (Thermo Fisher Scientific).

Western blot was also performed to detect the presence of flagellar proteins in the bacterial samples. The membrane was probed with 1:1,000 anti-E. coli Flagella H Pool A serum (Statens Serum Institut, Copenhagen, Denmark) as the primary antibody and 1:1,000 anti-rabbit IgG-AP antibody in TBST (Sigma-Aldrich, St Louis, MO, USA) as the secondary antibody. The intensity of the specific bands was visualized with a 1-step NBT/BCIP substrate solution (Thermo Fisher Scientific).

\section{Microscopy}

\section{Scanning electron microscopy}

Bacterial and BG samples for scanning electron microscopy (SEM) were fixed with 2.5\% glutaraldehyde in $0.06 \times$ PHEM buffer (1× PHEM buffer contains $60 \mathrm{mM}$ PIPES, $25 \mathrm{mM}$ HEPES, $10 \mathrm{mM}$ EGTA, and $2 \mathrm{mM} \mathrm{MgCl}_{2}$ ) and was prepared according to the previously described method ${ }^{25}$ and applied to $0.05 \%$ poly-L-lysine (Sigma-Aldrich)-coated glass cover slips, 
post-fixed in $1 \%$ aqueous osmium tetroxide (Agar Scientific, Stansted, UK), dehydrated in graded acetone, critical point dried in liquid $\mathrm{CO}_{2}$ (Leica CPD300, Leica Microsystems GmbH, Vienna, Austria), gold sputter-coated (Agar 108, Agar Scientific), and viewed with a Philips XL20 SEM at $20 \mathrm{kV}$.

\section{Immunofluorescence microscopy for flagella}

EcN and MOMP-EcN BGs were fixed with 4\% paraformaldehyde in $0.06 \times$ PHEM buffer to preserve external membrane structures. Aliquots of fixed bacterial cells or BGs $(120 \mu \mathrm{L})$ were processed as previously described ${ }^{26}$ with modifications described herein. Prior to antibody staining, the samples were pretreated with $1 \%$ bovine serum albumin (BSA) $-0.5 \%$ Triton X-100 for 10 minutes at room temperature (RT) to permeabilize the cells. Then, the cells were treated with $1 \%$ lysozyme (Sigma-Aldrich) in PBS for 15 minutes at RT. Samples were stained with anti-E. coli Flagella H Pool A serum at 1:100 in antibody diluent ( $2 \%$ BSA, $0.25 \%$ Triton X-100; Sigma-Aldrich). For secondary antibody staining, 1:500 goat anti-rabbit IgG labeled with Alexa Fluor 546 (Thermo Fisher Scientific) was used. Cell suspensions were applied to a $1 \%$ agarose-coated microscope slide ${ }^{27}$ and mounted with Vectashield fluorescent antibody mounting medium. The samples were viewed and imaged using a Nikon Eclipse 50i microscope equipped with an MFCool camera (Jenoptik, Jena, Germany). Images were acquired using the ProgRes Capture Pro 2.8.8 software (Jenoptik) and processed using the public domain program ImageJ. ${ }^{28}$

\section{Immunoelectron microscopy for flagella}

Drops of EcN or pBGKB-MOMP-EcN (pGLysivb) from 4\% formaldehyde and $0.06 \times$ PHEM-fixed samples were allowed to settle onto $0.05 \%$ poly-L-lysine (Sigma-Aldrich)-coated $0.5 \%$ formvar-support electron microscopy grids. Immunoelectron microscopy for flagella was performed as previously described. ${ }^{29}$ Anti-E. coli Flagella H Pool A serum diluted to $1: 100$ in antibody diluent (2\% BSA, $0.25 \%$ Triton X-100; Sigma-Aldrich) was used as the primary antibody, and 1:20 goat anti-rabbit IgG-12 nm or $6 \mathrm{~nm}$ colloidal gold-linked antibody (Jackson ImmunoResearch Europe Ltd., Newmarket, UK) was used as the secondary antibody. Sample grids were contrasted for electron microscopy by negative staining with aqueous $1 \%$ ammonium molybdate (Sigma-Aldrich) $)^{30}$ and examined with a Zeiss Libra 120 transmission electron microscope (TEM) at $120 \mathrm{kV}$. Images were recorded with a SharpEye camera system (Olympus Corporation, Tokyo, Japan) using the AnalySIS 5.0 program (Olympus Corporation) and processed using Photoshop CS6 and Illustrator CS6 (Adobe Systems, Inc., San Jose, CA, USA).

\section{Immunofluorescence microscopy for c-Myc expression}

EcN and MOMP-EcN BGs were fixed with 4\% paraformaldehyde in $0.06 \times$ PHEM buffer to preserve the external membrane structures. Cell pellets were washed in $1 \times$ PHEM buffer, dehydrated with $70 \%$ acetone for 2 and 5 minutes, followed by $100 \%$ acetone for $4 \times 5$ minutes before infiltration with pure LR White resin (Science Services GmbH, Munich, Bavaria, Germany). The pure resin was exchanged twice for 15 minutes before embedding in fresh pure LR white resin. Pellets were polymerized at $45^{\circ} \mathrm{C}$ for 48 hours. Sections with a thickness of $0.5 \mu \mathrm{m}$ were cut with Leica Ultracut 6 ultramicrotome (Leica Microsystems $\mathrm{GmbH}$ ) onto BioBond (BBI Solutions, Cardiff, UK)-coated glass slides. Sections were pretreated with $50 \mathrm{mM}$ glycine for 10 minutes and $1 \%$ trypsin in PBS for 10 minutes at RT before blocking with antibody diluent ( $2 \%$ BSA, $0.25 \%$ Triton X-100 in PBS). The primary antibodies of mouse monoclonal anti-c-Myc (1:50, BioLegend) and rabbit polyclonal anti-EcN antibody (1:250, produced in our laboratory) were applied simultaneously for 1 hour at $37^{\circ} \mathrm{C}$. The secondary antibodies of donkey antimouse Alexa Fluor 488 (Invitrogen, Life Technologies) and goat anti-rabbit Alexa Fluor 546 (Thermo Fisher Scientific) diluted to 1:500 in antibody diluent were applied simultaneously for 20 minutes at $37^{\circ} \mathrm{C}$. After washing, slides were mounted in Dako fluorescence mounting medium (Dako Denmark A/S, Glostrup, Denmark). Samples were viewed and imaged using a Nikon Eclipse 50i microscope equipped with an MFCool camera (Jenoptik). Images were acquired using the ProgRes Capture Pro 2.8.8 software (Jenoptik) and processed using the public domain program ImageJ. ${ }^{28}$

\section{Immunoelectron microscopy for c-Myc expression}

Immunoelectron microscopy was performed on ultrathin Lowicryl K11M sections of N-PmpC-EcN BGs. Bacterial pellets of pBGKB-N-PmpC EcN (pGLysivb), both before and after $E$-lysis, were fixed by high-pressure freezing (HP100, Leica Microsystems $\mathrm{GmbH}$ ) and freeze substituted in $100 \%$ dry acetone at $-90^{\circ} \mathrm{C}$ for 8 hours, and $-50^{\circ} \mathrm{C}$ for another 8 hours. Samples were infiltrated at $-50^{\circ} \mathrm{C}$ with Lowicryl K11M resin (Polysciences, Inc., Warrington, PA, USA) and then embedded in fresh resin and polymerized at $-50^{\circ} \mathrm{C}$ under UV irradiation (Leica AFS, Leica Microsystems $\mathrm{GmbH}$ ). Ultrathin sections were mounted onto formvarcoated nickel electron microscopy grids.

$\mathrm{N}-\mathrm{PmpC}$ expression in EcN was visualized using purified mouse anti-c-Myc antibody (1:200 dilution) (BioLegend) as the primary antibody, and donkey anti-rabbit IgG-10 nm gold colloid (1:30, Aurion, Utrecht, the Netherlands) was used as 
the secondary antibody. To enhance the ultrastructural visualization, labeled membranes were contrasted with $0.05 \%$ uranyl acetate (Leica Microsystems $\mathrm{GmbH}$ ) for 8 minutes at RT. Samples were examined with a Zeiss 902 TEM at $80 \mathrm{kV}$. Images were recorded with a SharpEye camera system (Olympus Corporation) using the AnalySIS 5.0 program (Olympus Corporation). Images were processed using Photoshop CS6 and Illustrator CS6 (Adobe Systems, Inc.).

\section{Epithelial cell culture}

Human conjunctival epithelial $(\mathrm{HCjE})$ cells were maintained in keratinocyte serum-free medium (Thermo Fisher Scientific) in $5 \% \mathrm{CO}_{2}$ as previously described. ${ }^{31}$

\section{Live cell imaging}

Atto488-labeled EcN and N-PmpC-EcN BGs, prepared as previously described, ${ }^{31}$ were applied (multiplicity of infection 1,000 BGs per cell) to $80 \%$ confluent $\mathrm{HCjE}$ cell cultures in glass bottom imaging plates (PAA Laboratories $\mathrm{GmbH}$, Pasching, Austria) and centrifuged at $315 \times \mathrm{g}$ for 15 minutes to enhance the initial attachment. Cells and BGs were incubated for an additional 105 minutes at $37^{\circ} \mathrm{C}$ in $5 \%$ $\mathrm{CO}_{2}$ before extensive washing to remove unattached BGs.

For visualization by epifluorescence microscopy, live cells were incubated with the plasma membrane stain CellMask (5 $\mathrm{gg} / \mathrm{mL}$; Molecular Probes Inc., Eugene, OR, USA) for 5 minutes at $37^{\circ} \mathrm{C} .4^{\prime}, 6$-Diamidino-2-phenylindole $(1 \mu \mathrm{g} / \mathrm{mL}$; Sigma-Aldrich) was used as a nuclear counterstain. Plates were mounted and examined by epifluorescence microscopy (Zeiss Axiovert 100, Carl Zeiss GmbH, Vienna, Austria).

\section{Results \\ $B G$ s retain their outer membrane surface structures after E-lysis}

Retention of the outer membrane surface structures is crucial to the attachment and uptake of EcN and imparts important immunomodulatory effects on host cells. Before further EcN BG development, we wanted to reassure that these outer membrane structures were retained after $E$-lysis. Therefore, we used electron microscopy to investigate whether BGs retained their fimbriae and flagella. Indeed, we illustrated in a set of SEM images that both fimbriae and flagella were present on both live EcN and EcN BGs (Figure 1). The small sample size examined with SEM precludes quantitation of flagella and fimbriae; however, both outer surface structures are present in abundance in both live EcN (Figure 1A) and $\mathrm{EcN}$ BGs (Figure 1B). The "hole" originating from the $E$-lysis of EcN was clearly visible on EcN BGs (Figure 1C
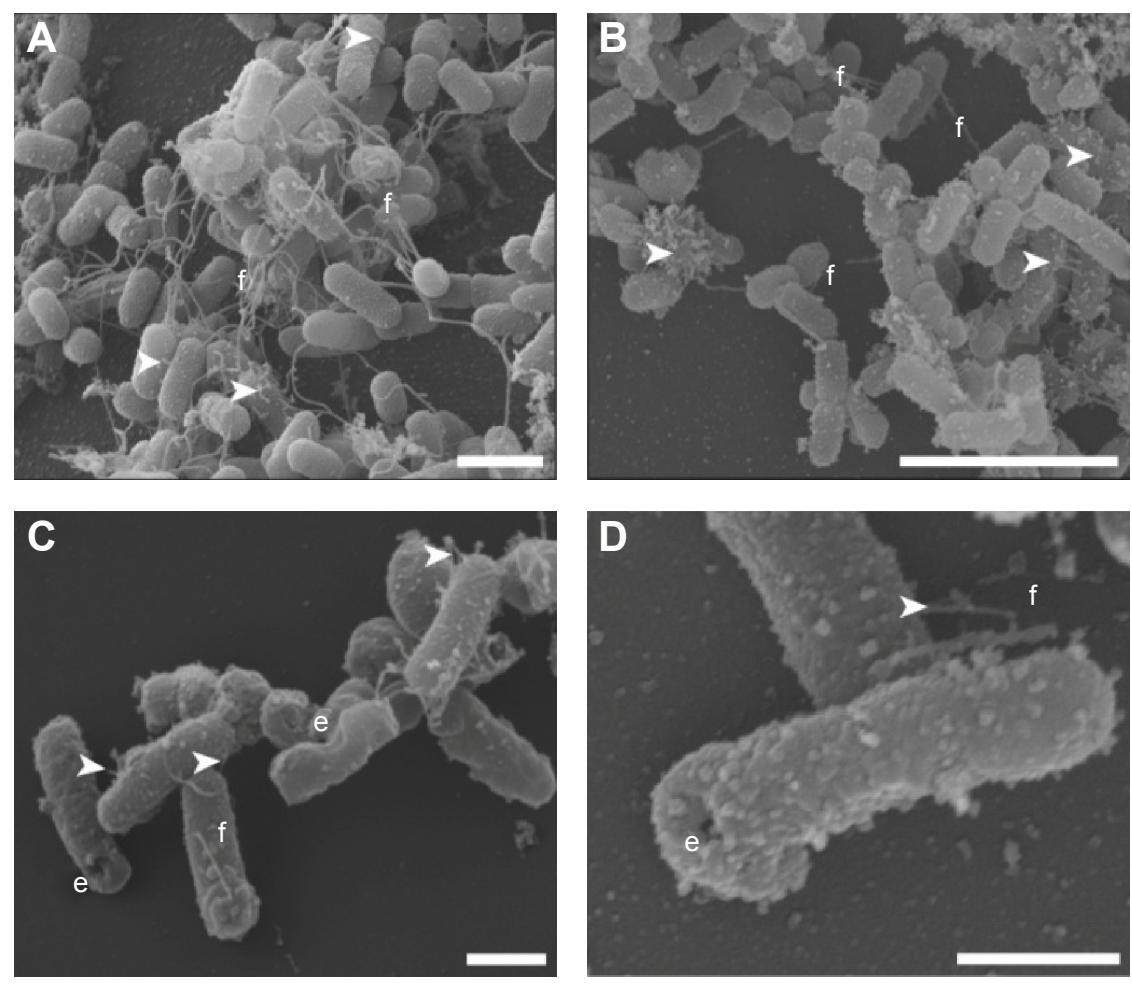

Figure I Scanning electron microscopic images, showing both EcN and EcN BGs expressing foreign chlamydial antigen.

Notes: $(\mathbf{A}) \mathrm{N}-\mathrm{PmpC}$-EcN during exponential growth showing abundant flagellae and fimbriae. (B, C, and $\mathbf{D}) \mathrm{N}$-PmpC-EcN BGs after E-lysis showing retention of flagellae (f) and fimbriae (white arrowheads). The E-lysis pore is visible (e) on some of the EcN BGs. Scale bars: (A) $2 \mu \mathrm{m}$; (B) $5 \mu \mathrm{m}$; (C) I $\mu \mathrm{m}$; and (D) $500 \mathrm{~nm}$.

Abbreviations: BGs, bacterial ghosts; EcN, Escherichia coli strain Nissle 1917; N-PmpC, N-terminal part of polymorphic membrane protein C. 
and D). Further confirmation that fimbriae are retained after $E$-lysis, is visualized by negative-contrast TEM, shown in Figure S1.

Using immunoelectron microscopy and immunohistochemistry (Figure 2), we demonstrated specific labeling of flagella by antibodies against $\mathrm{H} 1$ flagellin. The binding of $\mathrm{H}-\mathrm{Pool} \mathrm{A}$ antigens, which contain $\mathrm{H} 1$ in addition to other $\mathrm{H}$-antigens, enabled the identification of the long surface projections of EcN and EcN BGs as flagella. Flagella expression on live EcN (Figure 2A and B) was similar to flagella expression on EcN BGs (Figure 2C and D). The colloidal gold labeling of flagella structures both before (Figure 2E and $\mathrm{F}$ ), and after $E$-lysis (Figure $2 \mathrm{G}$ and $\mathrm{H}$ ) confirmed the identity of the flagella.

The expression of flagella was confirmed by Western blot prior to the induction of protein expression, after induction with L-arabinose and also after $E$-lysis (Figure 3 ). We attributed the fainter appearance of the flagella band after $E$-lysis to the expulsion of cytoplasmic flagellin proteins into the media, where they were lost during washing. Thus, the amounts of flagellin protein detected after $E$-lysis (lane C) reflect the amount of foreign proteins remaining on the BGs.

\section{Chlamydial outer membrane proteins are expressed on EcN BGs}

Expression of c-Myc- and poly-histidine-tagged chlamydial antigens after the induction of protein expression with
L-arabinose was confirmed via Western blot (Figure 4). The cell lysate pellets prepared for Western blot analysis were taken after removal of growth media (W1) and subsequently after PBS wash (W2), contained less protein than sequential samples taken before washing steps.

Retention of foreign-expressed proteins by the EcN BGs was verified with both immunofluorescence microscopy and immunoelectron microscopy (Figure 5). Expression of chlamydial MOMP was confirmed with immunofluorescence labeling of both the c-Myc-tagged MOMP and the E. coli outer membrane of the MOMP-EcN BGs (Figure 5A-E and G). Additionally, the expression of chlamydial N-PmpC was visualized inside the cytoplasmic compartments of the bacterial cells with the colloidal gold labeling in live N-PmpC-EcN (Figure 5E) and lysed N-PmpC-EcN BGs (Figure 5F-H).

Our results showed that before $E$-lysis expression, $\mathrm{N}-\mathrm{PmpC}$ was detected at the poles of live EcN, in an area of rapid growth or imminent cell division (Figure 5E). After $E$-lysis and expulsion of DNA and cytoplasmic elements, these same polar areas on the EcN BG exhibited the highest antigen expression. In addition, the Western blot results indicated no substantial difference between the amount of protein expression before and after $E$-lysis. Further evidence that the exponential growth of $\mathrm{EcN}$ was not impaired by the expression of foreign chlamydial antigens is shown by comparative bacterial growth curves in Figure S2.
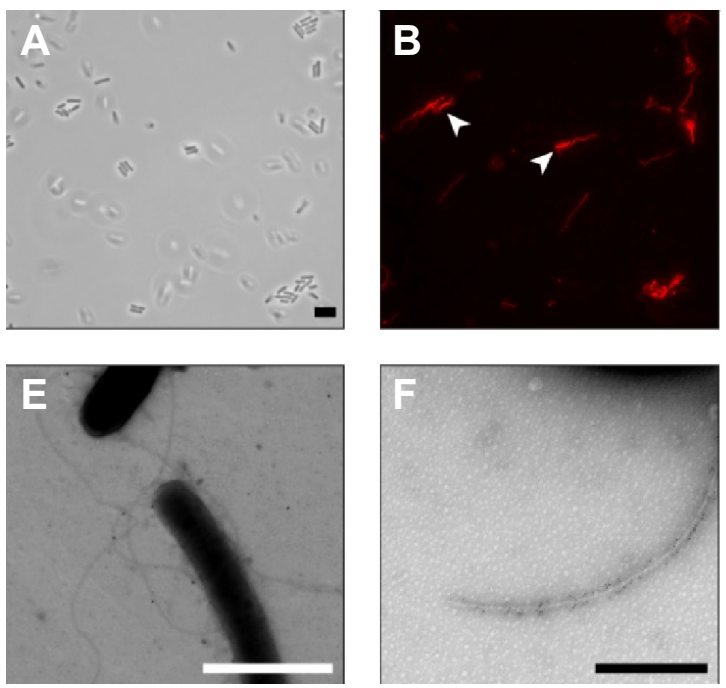
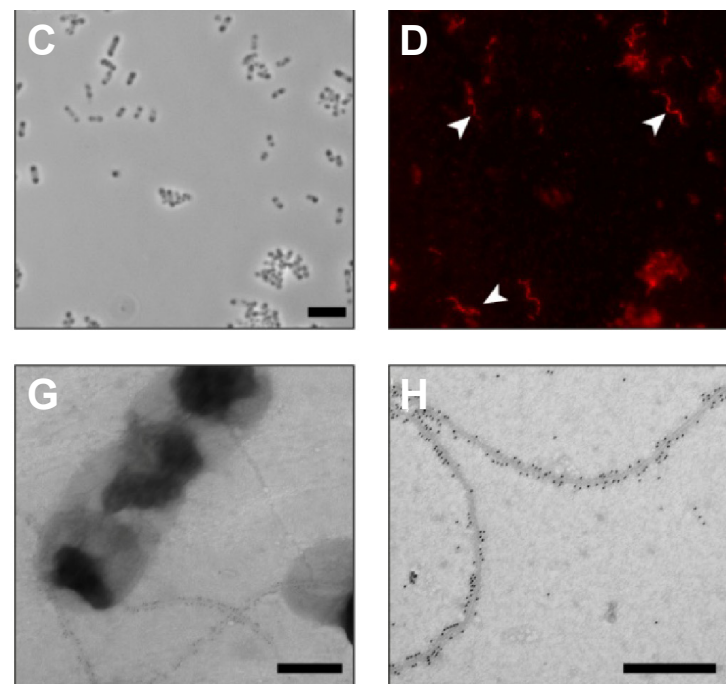

Figure 2 Flagella of both EcN and EcN BGs were identified using immunohistochemistry and immunoelectron microscopy.

Notes: (A) EcN (pBGKB-MOMP, pGLysivb) bacteria, transmitted light; (B) flagella of EcN (pBGKB-MOMP, pGLysivb) are localized and labeled with Alexa Fluor 546 (white arrowheads); (C) MOMP-EcN (pBGKB-MOMP, pGLysivb) BGs, transmitted light; (D) flagella of MOMP-EcN (pBGKB-MOMP, pGLysivb) BGs are localized and labeled with Alexa Fluor 546 (white arrowheads); (E) TEM image of EcN (pBGKB-MOMP, pGLysivb) bacteria, showing negatively contrasted flagella labeled with $25 \mathrm{~nm}$ colloidal gold; (F) high-magnification TEM image of EcN (pBGKB-MOMP, pGLysivb) bacteria, showing negatively contrasted flagella labeled with $6 \mathrm{~nm}$ colloidal gold; (G) lower magnification and $(\mathbf{H})$ high-magnification TEM image of MOMP-EcN (pBGKB-MOMP, pGLysivb) BGs showing negatively contrasted flagella labeled with 12 nm colloidal gold. Scale bars: (A-D) $5 \mu \mathrm{m}$; (E) $2 \mu \mathrm{m}$; and (F-H) $500 \mathrm{~nm}$.

Abbreviations: BGs, bacterial ghosts; EcN, Escherichia coli strain Nissle 1917; MOMP, major outer membrane protein; TEM, transmission electron microscope. 


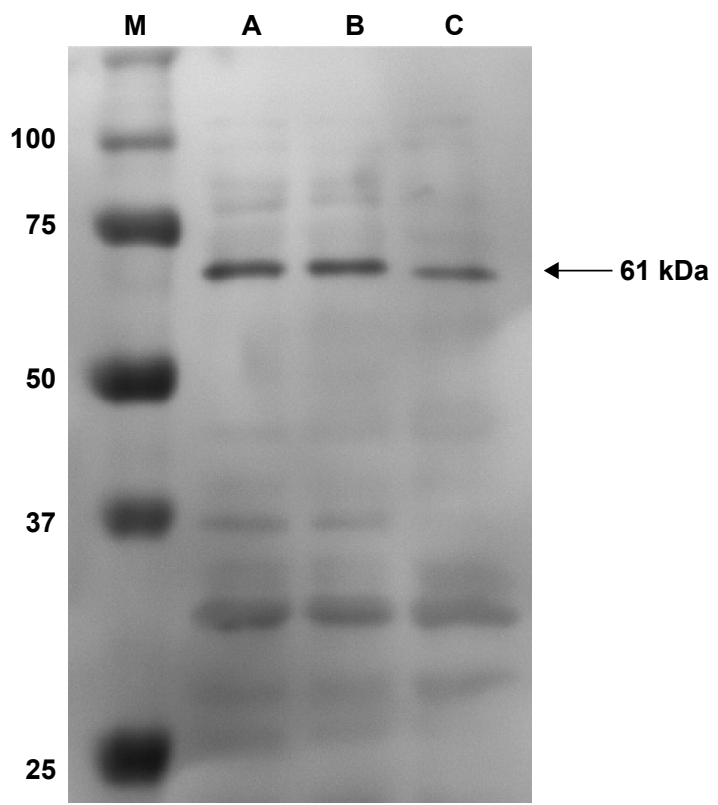

Figure 3 Cell extracts of EcN (pBGKB-MOMP, pGLysivb) from different time points were separated on a $4 \%-12 \%$ polyacrylamide gel and presence of Escherichia coli $\mathrm{HI}$-flagellin (MW $6 \mathrm{I}$ kDa) was visualized by Western blot.

Notes: Samples were taken at intervals during a $20 \mathrm{~L}$ batch fermentation, immediately before and 10-130 minutes after induction of protein expression with L-arabinose (lanes labeled A, B, and C, respectively). Lane M shows molecular weight marker. E-lysis was induced 10 minutes after addition of L-arabinose by increasing the temperature to $42^{\circ} \mathrm{C}$.

Abbreviations: EcN, Escherichia coli strain Nissle 1917; MOMP, major outer membrane protein; $\mathrm{MW}$, molecular weight.

\section{Expression of foreign chlamydial antigens in EcN BGs does not inhibit BG attachment to conjunctival cells in vitro}

Carriage of foreign antigens in the EcN BG could reduce their attachment to the conjunctival cells, which may then inhibit the subsequent BG uptake and processing of the foreign antigen by the immune cells. For these reasons, we used epifluorescence microscopy of $\mathrm{HCjE}$ cells co-incubated with EcN and N-PmpC EcN BGs to confirm the attachment of BGs. Figure 6 shows a representative picture of $\mathrm{EcN}$ (Figure 6A) and N-PmpC EcN BGs (Figure 6B) inside HCjE cells for 120 minutes after the application of 1,000 BGs per $\mathrm{HCjE}$ cell. This result showed that attachment by "armed"EcN BG occurred without detectable negative effects on HCjE cell viability.

\section{Discussion}

New drug-delivery systems to prevent and treat ocular surface diseases are needed, urgently. The treatment system that we are developing uses BGs derived from $\mathrm{EcN}$. The treatment of the ocular surface disease based on the use of probiotic eye drops was previously shown to be beneficial. ${ }^{2}$ This study describes the new development of a BG platform with the

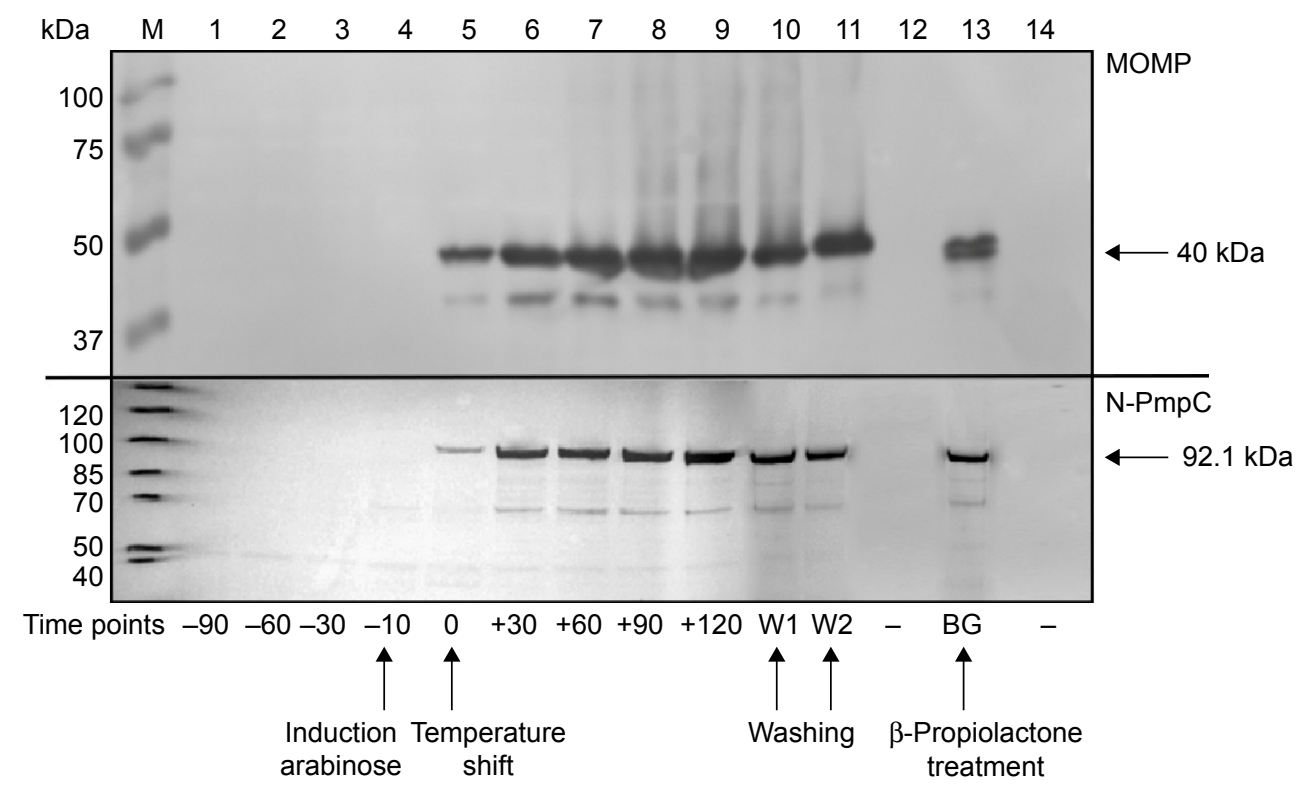

Figure 4 Cell lysates of EcN (pBGKB-MOMP, pGLysivb and pBGKB-N-PmpC, pGLysivb) were separated on a 4\%-12\% polyacrylamide gel and c-Myc-tagged MOMP and $\mathrm{N}-\mathrm{PmpC}$ protein levels were determined by Western blot.

Notes: Samples for lanes I-3 were taken at sequential intervals $(-90,-60$, and -30 minutes) during exponential growth. Lane $M$ shows molecular weight marker. Upon induction with L-arabinose (time point -10 minutes, lane 4) expression of MOMP and N-PmpC is induced. To induce $E$-lysis temperature is increased to $42^{\circ} \mathrm{C}$ (time point 0 minutes, lane 5). The process to complete transformation of live EcN to EcN BGs is consecutively monitored in 30 minutes intervals after induction of E-lysis (time point from 30 minutes to 120 minutes; lanes 6-9). After complete E-lysis, EcN BGs are washed and samples are taken after removal of growth media (WI) and subsequently after PBS wash (W2) (lanes I0 and II). Lanes 12 and 14 are negative controls; and lane 13 shows positive MOMP and N-PmpC expression after $\beta$-Propiolactone treatment to inactivate remnant nucleic acids.

Abbreviations: BGs, bacterial ghosts; EcN, Escherichia coli strain Nissle 1917; MOMP, major outer membrane protein; N-PmpC, N-terminal part of polymorphic membrane protein C; PBS, phosphate-buffered saline. 

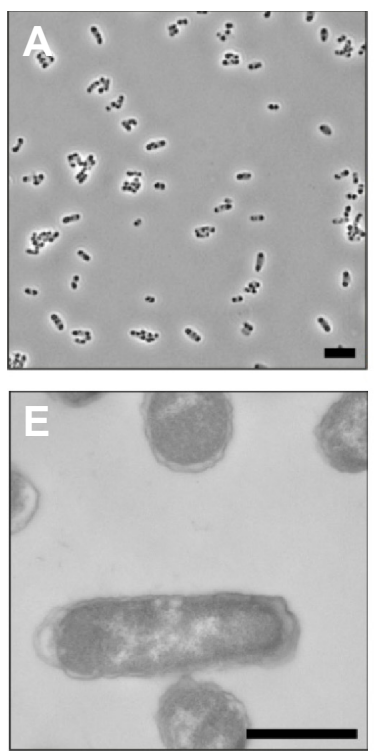
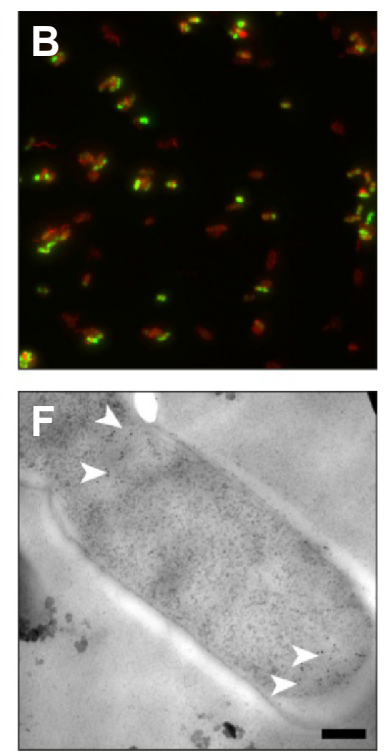
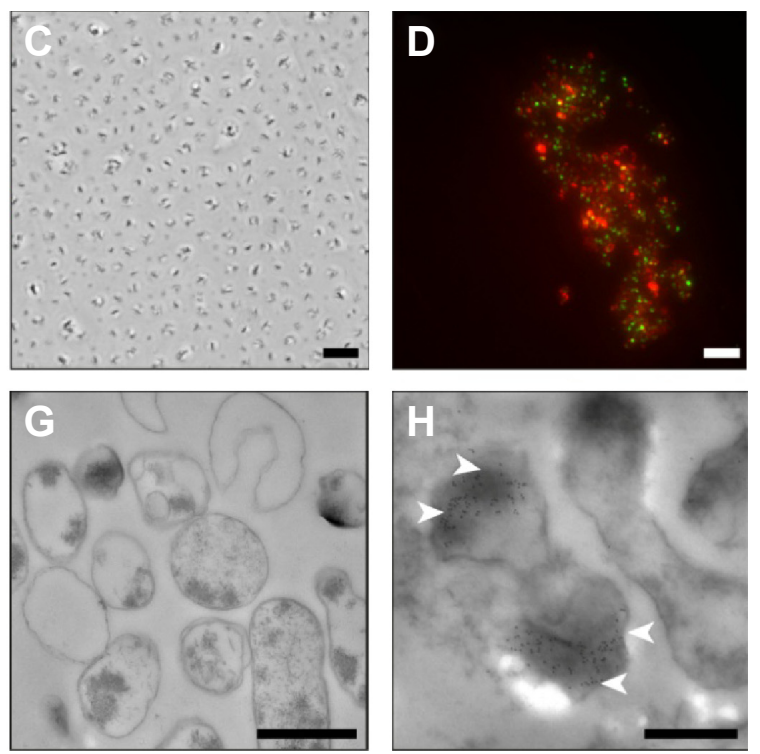

Figure 5 Microscopic confirmation of BGs expressing recombinant foreign chlamydial antigens.

Notes: (A) Whole EcN BGs (pBGKB-MOMP, pGLysivb) bacteria, transmitted light; (B) whole EcN (pBGKB-MOMP, pGLysivb) are localized and labeled with Alexa Fluor 546 (Escherichia coli outer membrane) and Alexa Fluor 488 (c-Myc-MOMP); (C) semi-thin sections of EcN BGs (pBGKB-MOMP, pGLysivb) are stained with toluidine blue, transmitted light; (D) expressed proteins of EcN BGs (pBGKB-MOMP, pGLysivb) are localized and labeled on semi-thin sections with Alexa Fluor 546 (E. coli outer membrane) and Alexa Fluor 488 (c-Myc-MOMP); (E) TEM image of EcN (pBGKB-N-PmpC, pGLysivb) bacteria; (F) TEM image of EcN BGs (pBGKB-N-PmpC, pGLysivb) bacteria; (G) TEM image of EcN BGs (pBGKB-N-PmpC, pGLysivb) bacteria; and (H) TEM image of N-PmpC-EcN BGs (pBGKB-N-PmpC, pGLysivb) showing c-Myc-N-PmpC expressed protein, labeled with $12 \mathrm{~nm}$ colloidal gold. The arrows in $(\mathbf{F})$ and $(\mathbf{H})$ show areas labeled with $10 \mathrm{~nm}$ colloidal gold particles, indicating $\mathrm{N}-\mathrm{PmpC}$ antigen coexpressed with c-Myc protein, in the bacterial cell (F) and also in the BG (H). Scale bars: (A-D) $5 \mu \mathrm{m} ;(\mathbf{E})$ I $\mu \mathrm{m} ;(\mathbf{F}) 200 \mathrm{~nm} ;(\mathbf{G})$ I $\mu \mathrm{m}$; and (H) $500 \mathrm{~nm}$.

Abbreviations: BGs, bacterial ghosts; EcN, Escherichia coli strain Nissle 1917; MOMP, major outer membrane protein; N-PmpC, N-terminal part of polymorphic membrane protein C; TEM, transmission electron microscope.

additional advantage of being a nonliving carrier-delivery system to increase both ocular safety and tolerability. ${ }^{32}$

The reason for treatment success using a live probiotic for keratoconjunctivitis vernalis was proposed to be similar to the effect observed in (the probiotic treatment of) allergic dermatitis: stimulation of the production of anti-inflammatory cytokines and immunomodulation via TLR stimulation. ${ }^{2}$ TLR stimulation by EcN, our chosen probiotic, is linked to the flagella and fimbriae surface structures on the bacterial membrane. Thus, to be as effective in immunomodulation as the live $\mathrm{EcN}$, the EcN BGs should retain their outer surface structures, such as fimbriae and flagella, which are both adhesins to human mucus. In particular, flagella have been identified to be crucial to the attachment and uptake of live EcN into epithelial cells. ${ }^{13}$ However, flagella could easily be broken off the bacterial cells during production of BGs.
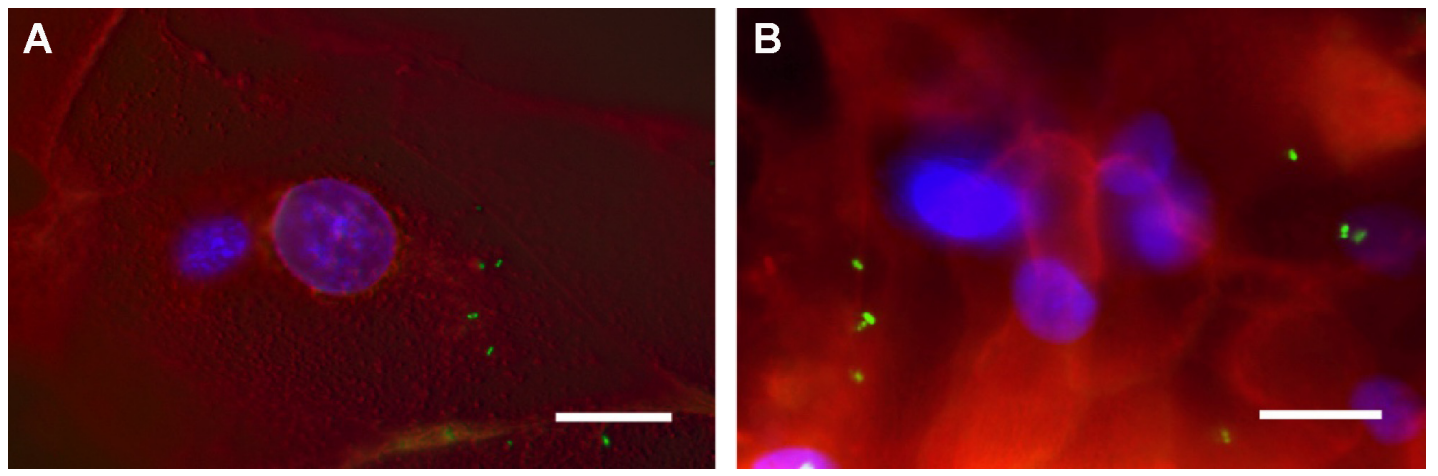

Figure 6 Analysis of Atto488-labeled EcN (pGLysivb) (A) and N-PmpC-EcN BGs (pBGKB-N-PmpC, pGLysivb) (B) attachment to HCjE cells by epifluorescence microscopy.

Notes: HCjE cells were incubated for 120 minutes with Atto488-EcN BGs ( I,000 per cell), washed with PBS, and cells on imaging plates were directly subjected to live cell staining. Scale bar: (A and B) $10 \mu \mathrm{m}$.

Abbreviations: BGs, bacterial ghosts; EcN, Escherichia coli strain Nissle 1917; HCjE, human conjunctival epithelial; N-PmpC, N-terminal part of polymorphic membrane protein C; PBS, phosphate-buffered saline. 
The presence of flagella was illustrated in live Helicobacter pylori prior to $E$-lysis, ${ }^{33}$ but they have not been identified by electron microscopy in BGs. Thus, it was important to examine whether these essential outer membrane structures were preserved after $\mathrm{EcN} B \mathrm{~B}$ production.

In our study, we confirmed that flagella were present on $\mathrm{EcN}$ both before and after $E$-lysis through a number of methods, including Western blot, immunofluorescence, SEM, and TEM.

The structural features observed in our study of thin sections of EcN BGs with electron microscopy closely resemble the appearance of $H$. pylori BGs noted in the previous work. ${ }^{33}$ Although the outer membranes were largely intact on $H$. pylori BGs, thin sections and negative staining images showed that not all BGs were completely empty, suggesting that the complete cytoplasmic content had not leaked out from every cell. ${ }^{33}$ However, when the $H$. pylori BGs were used for mouse mucosal vaccination studies, a protective effect was achieved. Hence, we did not expect that any small residue content would influence the effectiveness of the EcN BGs.

The importance of flagella to immunomodulation has been highlighted by recent studies showing that flagellin proteins have intrinsic effects that have been exploited for vaccine development. ${ }^{34}$ Flagellin has been shown to be a potent activator of a broad range of cell types involved in innate and adaptive immunity and is an effective adjuvant. ${ }^{35}$ Virus-like particles containing flagellin promoted typical responses of Th1 class immunity in BALB/c mice, as indicated by the enhanced production of IgG2a and $\operatorname{IgG} 2 \mathrm{~b}$ but not IgG1. The adjuvant effects of flagellin on specific cellular responses were observed in cytokine secretion and crossprotective immune responses against different subtypes. ${ }^{9}$ The powerful immunostimulatory properties of flagellin further improved immune responses toward co-administered protein antigens. ${ }^{36,37}$ Nevertheless, cell-associated flagella may confer an advantage over exported flagellin subunits because attenuated Salmonella vaccines strongly enhanced mucosal immunity protection. Although flagellin protein alone is a potent adjuvant for mucosal immunity, ${ }^{35}$ cell-associated polymerized flagella are preferable to free flagellin subunitbased immune system stimulators. ${ }^{38}$ Thus, the EcN BGs with cell-associated flagella should enhance their effectiveness as an immunomodulating delivery-treatment system.

$\mathrm{EcN}$ fimbriae are crucial for attachment to abiotic surfaces and cell surfaces in biofilm formation and intestinal colonization. ${ }^{39}$ In addition, fimbriae have been reported to be important for uptake of other E. coli strains in cells. ${ }^{40}$
Therefore, EcN fimbriae are predicated to be relevant for uptake in cells as well. The obtained SEM images did not allow for the identification of fimbriae types.

Chlamydial outer membrane proteins are difficult to generate as recombinant proteins. ${ }^{41}$ To overcome this limitation, we decided to express two different leaderless or N-terminal parts of CT outer membrane proteins, as model proteins for trachoma treatment, in the periplasmic space of EcN and generate "armed-BGs" from these bacteria. The EcN BGs were constructed to carry CT outer membrane proteins under control of the $\mathrm{pBAD}$ promoter, allowing for the induction of foreign protein expression with L-arabinose. These cloned antigens were co-expressed as fusion proteins with a c-Myc-epitope and a poly-histidine tag downstream of the protein. We chose two different chlamydial outer membrane proteins as models for expressing foreign antigen in $\mathrm{EcN}$. Both MOMP and PmpC were used because they are immunogenic and expressed on the outer surface of chlamydial bacteria. As this bacteria cause trachoma, delivery of this foreign protein may be beneficial in the future treatment of this ocular surface disease. Expressed foreign chlamydial antigens are not lost during the production of the BGs. We proposed that the fainter bands in later time points are due to the washing process. Samples taken before washing also contained expelled (during E-lysis) cytoplasmic proteins, including cytoplasmic flagellin, and washing removed these proteins from the samples. Thus, we assumed that amounts of foreign protein detected after the washing step (W2) reflect amounts of foreign proteins remaining in the BGs.

The mucosal delivery of an immunogen involves attachment followed by cellular uptake of the protein or particle by endocytosis and finally immunogenic processing. The initial step during attachment is the binding of a ligand to a receptor on the cell surface. The $\mathrm{HCjE}$ cell line expresses various mammalian innate immune TLR proteins in vitro, including toll-like receptor 5 (TLR5) (Figure S3). TLR5 is known to specifically recognize flagella and stimulate mucus production, ${ }^{42}$ whereas other TLRs bind fimbriae. ${ }^{43,44}$ Other TLRs stimulated by contact with whole dead E. coli $(\mathrm{EcN})$ bacteria reversed gut defense impairment by antibiotic treatment, suggesting that in addition to the flagella, the intact membrane of the $\mathrm{BG}$ is beneficial for immune protection via non-defensin protein expression. ${ }^{45,46}$ The presence of flagella and fimbriae on EcN BGs confirms their ability to enhance immunomodulation ${ }^{1,47}$ via TLR5 signaling, causing the production of chemokines and cytokines that direct inflammation and induce adaptive immune responses. The attachment of the BGs to the conjunctival cells is a prerequisite for the 
uptake and internalization of bacteria. Our results demonstrated that the expression of foreign chlamydial N-PmpC within EcN BGs did not inhibit the attachment to $\mathrm{HCjE}$ cells. This conclusion agrees with data obtained from previous in vitro studies concluding that BGs produced from different bacterial origins represent a safe vaccine and drug delivery platform. ${ }^{7,48-51}$ Although the attachment of MOMP-EcN BGs to $\mathrm{HCjE}$ cells was not performed, we suggest that the results are likely to be similar.

BGs have several advantages over live-attenuated probiotics, as they address any safety risks of using live bacteria as a delivery system. ${ }^{52}$ For ocular conjunctival delivery, a number of different BGs were successfully taken up by the ocular surface epithelia without signs of toxicity and/or genotoxicity. ${ }^{5,31}$ BGs are straightforward to produce, costeffective and require no special storage conditions aside from freeze-drying to maintain their potency for years. ${ }^{5,7,14}$ The probiotic EcN BG would form an easy-to-produce GMP compatible delivery system with innate immune system-recognized surface structures with intrinsic immunomodulatory effects. ${ }^{16}$ Lacking any ability to infect or colonize, EcN BGs form an elegant and safe method of drug or antigen delivery to the ocular mucosal surface.

Further experiments, which would include protein quantitation in different cell compartments (cytoplasmic and periplasmic supernatant fractions of live bacteria, cell lysates, and supernatant of BGs) and the assessment of antigen stability, are necessary for the development of future clinical application of BGs. Foreign-modified chlamydial proteins, such as N-PmpC and MOMP, are important outer membrane components of CT, our model ocular pathogen. Showing that chlamydial outer membrane protein antigens expressed on EcN BGs is an important contribution to the projected future use of EcN BGs as a delivery system to enhance and promote ocular mucosal immunity against trachoma. Several immunogenic proteins could potentially be co-expressed such that immunogenic stimulation could be delivered via the conjunctiva-associated mucosa lymphoid tissue. ${ }^{14,15}$ This could be a further treatment use of BGs to stimulate both the innate and mucosal adaptive immune responses. An additional effect of probiotic treatment observed in patients with chronic gut inflammation is the rebalancing of the mucosal flora microbiome. ${ }^{10,53}$ This idea of probiotics modulating the immune response via conjunctival delivery would be consistent with the concept of the ocular surface being part of the extended mucosa-associated lymphoid tissue system. ${ }^{54}$

Together, these results are an important contribution to the projected future use of EcN BGs as a foreign antigen carrier and delivery system to enhance and promote ocular mucosal immunity.

\section{Conclusion}

Our results demonstrated that EcN BGs expressing chlamydial foreign antigens retain the desired structural membrane features. This work builds the foundation for further development of a drug and immunomodulatory delivery system, which is envisaged for future use as a therapeutic treatment or vaccine strategy against ocular surface diseases.

\section{Acknowledgments}

This research was funded by the "Laura Bassi Centres of Expertise" program of the Austrian Federal Ministry of Economy through the Austrian Research Promotion Agency (FFG project number 822768). The continuous cell line $\mathrm{HCjE}$ cells were a kind donation from Professor Ilene Gipson (Schepens Eye Research Institute, Harvard Medical School, Boston, MA). We acknowledge Professor Irene Lichtscheidl, Professor Waltraud Klepal, and Professor Jasna Strus for their helpful discussions. We thank Daniela Gruber and the Core Facility of Cell Imaging and Ultrastructure Research at the University of Vienna for the use of their facilities. We also thank Professor Lothar Beutin and Professor Matthew Laue (Robert Koch Institute, Berlin) for their helpful advice on Flagella $\mathrm{H}$ antigens, as well as Professor Heinz Schwarz (Max Plank Institute for Developmental Biology, Tübingen) for providing $6 \mathrm{~nm}$ and $12 \mathrm{~nm}$ colloidal gold-labeled secondary antibodies.

\section{Author contributions}

All authors contributed toward data analysis, drafting and critically revising the paper and agree to be accountable for all aspects of the work.

\section{Disclosure}

Werner Lubitz is the owner of BIRD-C and Ulrike Beate Mayr is an employee of BIRD-C, the company producing Bacterial Ghosts. The authors report no other conflicts of interest in this work.

\section{References}

1. Ueta M, Kinoshita S. Ocular surface inflammation is regulated by innate immunity. Prog Retin Eye Res. 2012;31(6):551-575.

2. Iovieno A, Lambiase A, Sacchetti M, Stampachiacchiere B, Micera A, Bonini S. Preliminary evidence of the efficacy of probiotic eye-drop treatment in patients with vernal keratoconjunctivitis. Graefes Arch Clin Exp Ophthalmol. 2008;246(3):435-441.

3. Gronbach K, Eberle U, Muller M, et al. Safety of probiotic Escherichia coli strain Nissle 1917 depends on intestinal microbiota and adaptive immunity of the host. Infect Immun. 2010;78(7):3036-3046. 
4. Olier M, Marcq I, Salvador-Cartier C, et al. Genotoxicity of Escherichia coli Nissle 1917 strain cannot be dissociated from its probiotic activity. Gut Microbes. 2012;3(6):501-509.

5. Kudela P, Koller VJ, Mayr UB, Nepp J, Lubitz W, Barisani-Asenbauer T. Bacterial ghosts as antigen and drug delivery system for ocular surface diseases: effective internalization of bacterial ghosts by human conjunctival epithelial cells. J Biotechnol. 2011;153(3-4):167-175.

6. Paukner S, Stiedl T, Kudela P, Bizik J, Al Laham F, Lubitz W. Bacterial ghosts as a novel advanced targeting system for drug and DNA delivery. Expert Opin Drug Deliv. 2006;3(1):11-22.

7. Mayr UB, Walcher P, Azimpour C, Riedmann E, Haller C, Lubitz W. Bacterial ghosts as antigen delivery vehicles. Adv Drug Deliv Rev. 2005; 57(9):1381-1391.

8. Muhammad A, Champeimont J, Mayr UB, Lubitz W, Kudela P. Bacterial ghosts as carriers of protein subunit and DNA-encoded antigens for vaccine applications. Expert Rev Vaccines. 2012;11:97-116.

9. Simon R, Curtis B, Deumic V, et al. A scalable method for biochemical purification of Salmonella flagellin. Protein Expr Purif. 2014;102 $1-7$.

10. Sonnenborn U, Schulze J. The non-pathogenic Escherichia coli strain Nissle 1917 - features of a versatile probiotic. Microb Ecol Health Dis. 2009;21(3-4):122-158.

11. Lebeer S, Vanderleyden J, De Keersmaecker SCJ. Host interactions of probiotic bacterial surface molecules: comparison with commensals and pathogens. Nat Rev Microbiol. 2010;8(3):171-184.

12. Schlee M, Wehkamp J, Altenhoefer A, Oelschlaeger TA, Stange EF, Fellermann K. Induction of human beta-defensin 2 by the probiotic Escherichia coli Nissle 1917 is mediated through flagellin. Infect Immun. 2007;75(5):2399-2407.

13. Troge A, Scheppach W, Schroeder BO, et al. More than a marine propeller - the flagellum of the probiotic Escherichia coli strain Nissle 1917 is the major adhesin mediating binding to human mucus. Int J Med Microbiol. 2012;302(7-8):304-314.

14. Eko FO, Barisani-Asenbauer T, Lubitz W. Development of a Chlamydia trachomatis bacterial ghost vaccine to fight human blindness. Hum Vaccin. 2008;4(3):176-183.

15. Barisani-Asenbauer T, Inic-Kanada A, Belij S, et al. The ocular conjunctiva as a mucosal immunization route: a profile of the immune response to the model antigen tetanus toxoid. PLoS One. 2013;8(4):e60682.

16. Jalava K, Eko FO, Riedmann E, Lubitz W. Bacterial ghosts as carrier and targeting systems for mucosal antigen delivery. Expert Rev Vaccines. 2003;2(1):45-51.

17. Fahmy AM, Hardten DR. Treating ocular surface disease: new agents in development. Clin Ophthalmol. 2011;5:465-472.

18. Becker E, Hegemann JH. All subtypes of the Pmp adhesin family are implicated in chlamydial virulence and show species-specific function. Microbiologyopen. 2014;3(4):544-556.

19. Tan C, Hsia R, Shou H, Carrasco J, Rank R, Bavoil P. Variable expression of surface-exposed polymorphic membrane proteins in in vitrogrown Chlamydia trachomatis. Cell Microbiol. 2010;12(2):174-187.

20. Teng A, Cruz-Fisher M, Cheng C, et al. Proteomic identification of immunodominant chlamydial antigens in a mouse model. J Proteomics. 2012;77:176-186.

21. Yu H, Karunakaran KP, Jiang X, Brunham RC. Evaluation of a multisubunit recombinant polymorphic membrane protein and major outer membrane protein T cell vaccine against Chlamydia muridarum genital infection in three strains of mice. Vaccine. 2014;32(36):4672-4680.

22. Zhu S, Chen J, Zheng M, et al. Identification of immunodominant linear B-cell epitopes within the major outer membrane protein of Chlamydia trachomatis. Acta Biochim Biophys Sin (Shanghai). 2010;42(11): 771-778.

23. Langemann T, Koller VJ, Muhammad A, Kudela P, Mayr UB, Lubitz W. The bacterial ghost platform system: production and applications. Bioeng Bugs. 2010;1(5):326-336.

24. Harlow E, Lane D. Antibodies: A Laboratory Manual. Cold Spring Harbor (NY): Cold Spring Harbor Laboratory Press; 1988.

25. Schliwa M, van Blerkom J. Structural interaction of cytoskeletal components. J Cell Biol. 1981;90(1):222-235
26. van der Ploeg R, Verheul J, Vischer NO, et al. Colocalization and interaction between elongasome and divisome during a preparative cell division phase in Escherichia coli. Mol Microbiol. 2013;87(5): 1074-1087.

27. Koppelman CM, Aarsman ME, Postmus J, et al. R174 of Escherichia coli FtsZ is involved in membrane interaction and protofilament bundling, and is essential for cell division. Mol Microbiol. 2004;51(3): 645-657.

28. Schneider CA, Rasband WS, Eliceiri KW. NIH Image to ImageJ: 25 years of image analysis. Nat Methods. 2012;9(7):671-675.

29. Beutin L, Strauch E, Zimmermann S, et al. Genetical and functional investigation of fliC genes encoding flagellar serotype $\mathrm{H} 4$ in wildtype strains of Escherichia coli and in a laboratory E. coli K-12 strain expressing flagellar antigen type H48. BMC Microbiol. 2005;5:4.

30. Hoppert M, Holzenburg A. Electron Microscopy in Microbiology. Vol 43. Oxford: Bios Scientific Publishers Ltd.; 1998.

31. Stein E, Inic-Kanada A, Belij S, et al. In vitro and in vivo uptake study of Escherichia coli Nissle 1917 bacterial ghosts: cell-based delivery system to target ocular surface diseases. Invest Ophthalmol Vis Sci. 2013;54(9): 6326-6333.

32. Taverniti V, Guglielmetti S. The immunomodulatory properties of probiotic microorganisms beyond their viability (ghost probiotics: proposal of paraprobiotic concept). Genes Nutr. 2011;6(3): 261-274.

33. Panthel K, Jechlinger W, Matis A, et al. Generation of Helicobacter pylori ghosts by PhiX protein E-mediated inactivation and their evaluation as vaccine candidates. Infect Immun. 2003;71(1):109-116.

34. Kumar A, Hazlett LD, Yu FS. Flagellin suppresses the inflammatory response and enhances bacterial clearance in a murine model of Pseudomonas aeruginosa keratitis. Infect Immun. 2008;76(1):89-96.

35. Mizel SB, Bates JT. Flagellin as an adjuvant: cellular mechanisms and potential. J Immunol. 2010;185(10):5677-5682.

36. Nart P, Holden N, McAteer SP, et al. Mucosal antibody responses of colonized cattle to Escherichia coli O157-secreted proteins, flagellin, outer membrane proteins and lipopolysaccharide. FEMS Immunol Med Microbiol. 2008;52(1):59-68.

37. Wang BZ, Quan FS, Kang SM, Bozja J, Skountzou I, Compans RW. Incorporation of membrane-anchored flagellin into influenza virus-like particles enhances the breadth of immune responses. J Virol. 2008;82(23): 11813-11823.

38. Gat O, Galen JE, Tennant S, et al. Cell-associated flagella enhance the protection conferred by mucosally-administered attenuated Salmonella paratyphi A vaccines. PLoS Negl Trop Dis. 2011;5(11):e1373.

39. Lasaro MA, Salinger N, Zhang J, et al. F1C fimbriae play an important role in biofilm formation and intestinal colonization by the Escherichia coli commensal strain Nissle 1917. Appl Environ Microbiol. 2009;75(1): 246-251.

40. Li K, Zhou W, Hong Y, Sacks SH, Sheerin NS. Synergy between type 1 fimbriae expression and $\mathrm{C} 3$ opsonisation increases internalisation of $E$. coli by human tubular epithelial cells. BMC Microbiol. 2009;9:64.

41. Longbottom D. Chlamydial vaccine development. J Med Microbiol. 2003;52(Pt 7):537-540.

42. Smith KD, Andersen-Nissen E, Hayashi F, et al. Toll-like receptor 5 recognizes a conserved site on flagellin required for protofilament formation and bacterial motility. Nat Immunol. 2003;4(12):1247-1253.

43. Li J, Shen J, Beuerman RW. Expression of toll-like receptors in human limbal and conjunctival epithelial cells. Mol Vis. 2007;13:813-822.

44. Tong L, Diebold Y, Calonge M, Gao J, Stern ME, Beuerman RW. Comparison of gene expression profiles of conjunctival cell lines with primary cultured conjunctival epithelial cells and human conjunctival tissue. Gene Expr. 2009;14(5):265-278.

45. Chen LW, Chen PH, Fung CP, Hsu CM. Dead bacteria reverse antibioticinduced host defense impairment in burns. J Am Coll Surg. 2014;219(4) 606-619.

46. Wu YY, Hsu CM, Chen PH, Fung CP, Chen LW. Toll-like receptor stimulation induces nondefensin protein expression and reverses antibiotic-induced gut defense impairment. Infect Immun. 2014;82(5): 1994-2005. 
47. Akira $\mathrm{S}$, Uematsu $\mathrm{S}$, Takeuchi O. Pathogen recognition and innate immunity. Cell. 2006;124(4):783-801.

48. Chaudhari AA, Jawale CV, Kim SW, Lee JH. Construction of a Salmonella Gallinarum ghost as a novel inactivated vaccine candidate and its protective efficacy against fowl typhoid in chickens. Vet Res. 2012;43:44.

49. Ekong EE, Okenu DN, Mania-Pramanik J, et al. A Vibrio cholerae ghost-based subunit vaccine induces cross-protective chlamydial immunity that is enhanced by CTA2B, the nontoxic derivative of cholera toxin. FEMS Immunol Med Microbiol. 2009;55(2):280-291.

50. Tu FP, Chu WH, Zhuang XY, Lu CP. Effect of oral immunization with Aeromonas hydrophila ghosts on protection against experimental fish infection. Lett Appl Microbiol. 2010;50(1):13-17.
51. Vilte DA, Larzabal M, Mayr UB, et al. A systemic vaccine based on Escherichia coli $\mathrm{O} 157: \mathrm{H} 7$ bacterial ghosts (BGs) reduces the excretion of E. coli O157:H7 in calves. Vet Immunol Immunopathol. 2012;146(2): 169-176.

52. Eko FO, Okenu DN, Singh UP, He Q, Black C, Igietseme JU. Evaluation of a broadly protective Chlamydia-cholera combination vaccine candidate. Vaccine. 2011;29(21):3802-3810.

53. Henker J, Laass MW, Blokhin BM, et al. Probiotic Escherichia coli Nissle 1917 versus placebo for treating diarrhea of greater than 4 days duration in infants and toddlers. Pediatr Infect Dis J. 2008;27(6):494-499.

54. Knop N, Knop E. Conjunctiva-associated lymphoid tissue in the human eye. Invest Ophthalmol Vis Sci. 2000;41(6):1270-1279. 


\section{Supplementary materials}
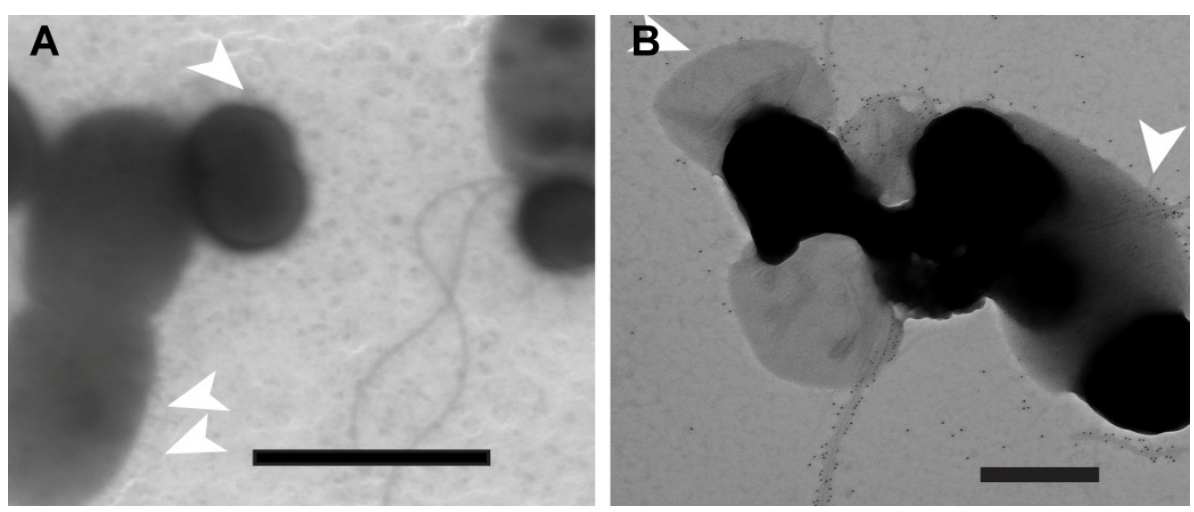

Figure SI Fimbriae are present on the surface of "armed"-EcN BGs.

Notes: TEM images of negatively stained EcN BGs showing fimbriae (white arrowheads) and flagella on the surface of (A) BGs expressing N-PmpC pGLysivb EcN; magnification scale bar $=2 \mu \mathrm{m}$ and (B) BGs expressing pBGKB-MOMP pGLysivb EcN; magnification scale bar $=500 \mathrm{~nm}$.

Abbreviations: BGs, bacterial ghosts; EcN, Escherichia coli strain Nissle 1917; MOMP, major outer membrane protein; N-PmpC, N-terminal part of polymorphic membrane protein C; TEM, transmission electron microscope.
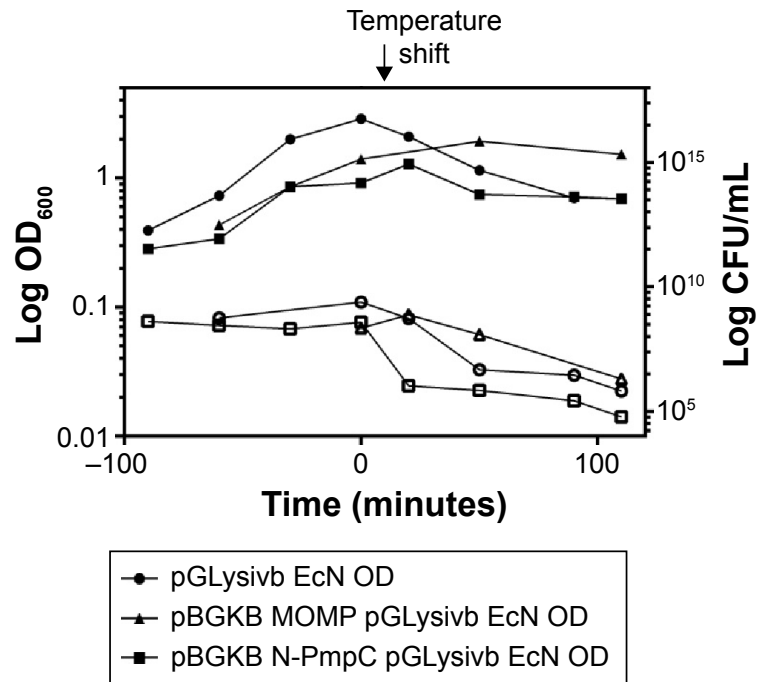

- pGLysivb EcN CFU

$\triangle$ pBGKB MOMP pGLysivb EcN CFU

๑ pBGKB N-PmpC pGLysivb EcN CFU

Figure S2 Growth curves during BG fermentation production.

Notes: Growth and lysis kinetics during fermentation comparing pGLysivb EcN, pBGKB-MOMP pGLysivb EcN, and pBGKB-N-PmpC pGLysivb EcN. Growth and lysis were monitored by measuring optical density at $600 \mathrm{~nm}\left(\mathrm{OD}_{600}\right)$ and viable cell counts were performed using a spiral plater to determine the number of $\mathrm{CFU} / \mathrm{mL}$. The arrow indicates the increase of temperature from $36^{\circ} \mathrm{C}$ to $42^{\circ} \mathrm{C}$ to initiate $E$-lysis.

Abbreviations: BG, bacterial ghost; CFU, colony-forming unit; EcN, Escherichia coli strain Nissle 1917; MOMP, major outer membrane protein; N-PmpC, N-terminal part of polymorphic membrane protein $\mathrm{C}$. 


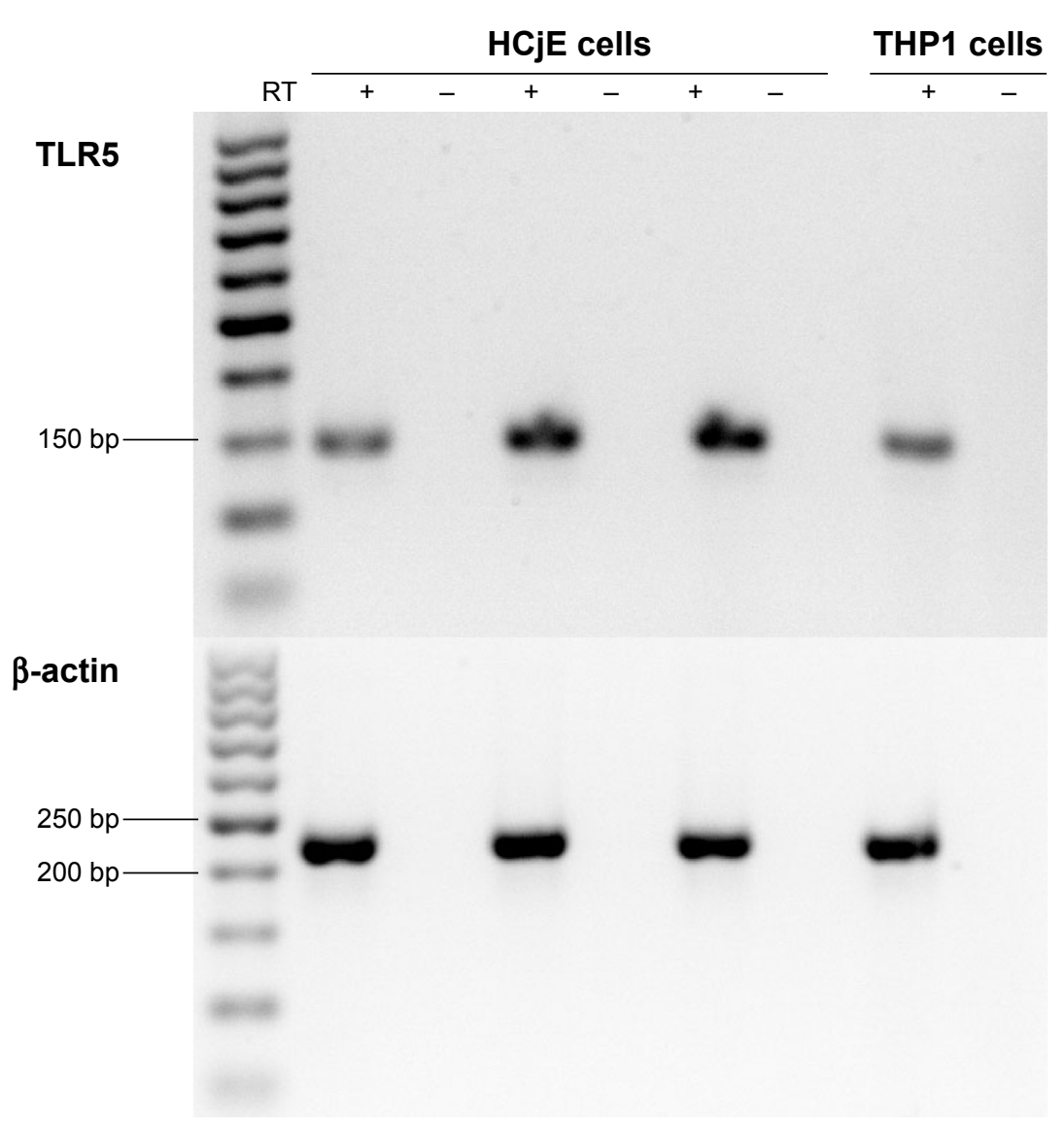

Figure S3 Toll-like receptor 5 (TLR5) is present in $\mathrm{HCjE}$ cells.

Notes: The expression of TLR5 and $\beta$-actin in HCjE. Three independent samples of untreated HCjE were taken and RNA was isolated by using RNeasy Mini Kit (Qiagen NV, Venlo, the Netherlands). This was followed by a DNase I treatment and RT-PCR with ThermoScript RT-PCR Kit (Invitrogen). RT-negative controls were included (RT-). PCR with 40 cycles was performed using TLR5 QuantiTect Primer Assay (Qiagen NV) and $\beta$-actin primers (fwd 5'GCACTCTTCCAGCCTTCCTT3', rev 5'CGCTCAGGAGGAGCAATGAT3') in a QuantiFast SYBR Green PCR Mastermix (Qiagen NV). THPI cells served as a positive control. PCR products for TLR5 (I47 bp) and $\beta$-actin (227 bP) were separated on a $2 \%$ agarose gel stained with GelRed (Qiagen, Hilden, Germany).

Abbreviations: $\mathrm{HCjE}$, human conjunctival epithelial; PCR, polymerase chain reaction; RT-PCR, reverse transcription polymerase chain reaction.

\section{Publish your work in this journal}

Drug Design, Development and Therapy is an international, peerreviewed open-access journal that spans the spectrum of drug design and development through to clinical applications. Clinical outcomes, patient safety, and programs for the development and effective, safe, and sustained use of medicines are a feature of the journal, which has also been accepted for indexing on PubMed Central. The manuscript management system is completely online and includes a very quick and fair peer-review system, which is all easy to use. Visit http://www.dovepress.com/testimonials.php to read real quotes from published authors.

Submit your manuscript here: http://www.dovepress.com/drug-design-development-and-therapy-journal 